\title{
ULRICH LINE BUNDLES ON DOUBLE PLANES
}

\author{
A. J. PARAMESWARAN AND POORNAPUSHKALA NARAYANAN
}

\begin{abstract}
Consider a smooth complex surface $X$ which is a double cover of the projective plane $\mathbb{P}^{2}$ branched along a smooth curve of degree $2 s$. In this article, we study the geometric conditions which are equivalent to the existence of Ulrich line bundles on $X$ with respect to this double covering. Also, for every $s \geq 1$, we describe the classes of such surfaces which admit Ulrich line bundles and give examples.
\end{abstract}

\section{INTRODUCTION}

Suppose that $X \stackrel{i}{\hookrightarrow} \mathbb{P}^{N}$ is a smooth projective variety over the field of complex numbers. A vector bundle $E$ on $X$ is said to be Ulrich if for any finite linear projection $\pi: X \rightarrow \mathbb{P}^{\operatorname{dim} X}$ obtained from $i$, the direct image $\pi_{*} E$ is the trivial vector bundle on the projective space, cf. $\S 2.1$ for equivalent definitions.

The study of Ulrich bundles has been an active area of research, especially since the paper of Eisenbud and Schreyer [10]. One of the primary questions in this area is that of the existence of Ulrich bundles on smooth projective varieties. In particular, one would also like to know the minimal rank of such a bundle whenever it exists. The existence of Ulrich bundles has been established in several cases e.g. when $X$ is a curve [10], a smooth complete intersection variety [16], an abelian surface [5], a general K3 surface [2], a grassmannian variety [8], a Fano threefold of index two [6], a ruled surface [1] etc.

In this article, we investigate the existence of Ulrich bundles on double planes. A double plane, in our context is a smooth projective surface $X$ which is a double cover of the projective plane $\mathbb{P}^{2}$. Such a surface has to necessarily be branched over a smooth even degree curve in $\mathbb{P}^{2}$. Refer $\S 2.2$ for some well-known details about double planes.

First, we identify the necessary and sufficient conditions for a double plane $\pi: X \rightarrow \mathbb{P}^{2}$ to carry an Ulrich line bundle with respect to $\pi$ (i.e. a line bundle $L$ on $X$ such that $\left.\pi_{*} L=\mathcal{O}_{\mathbb{P}^{2}} \oplus \mathcal{O}_{\mathbb{P}^{2}}\right)$ in the following

Theorem 1.1. Suppose that $\pi: X \rightarrow \mathbb{P}^{2}$ is a double plane branched over a smooth curve $B$ of degree $2 s$. Let $R$ denote the smooth ramified curve in $X$ and $\sigma$ denote the involution of $X$ obtained by interchanging the two sheets of the double cover. Then, the following are equivalent.

(1) The surface $X$ admits an Ulrich line bundle with respect to $\pi$.

(2) There is a smooth curve $D \subset X$ such that $D \neq \sigma(D)$ and $D \cdot \sigma(D)=D \cdot R=$ $\sigma(D) \cdot R=s^{2}$.

(3) There is a smooth curve $C$ of degree $s$ in $\mathbb{P}^{2}$ which is a tangent to $B$ of even order at every point of $C \cap B$.

Key words and phrases. Ulrich bundles, double planes, cyclic coverings.

Mathematics Classification numbers: 14E20, 14J60, 14H50, 14C20. 
We prove that conditions (1) and (2) are equivalent and they imply condition (3) in $\S 3$. We then show that (3) implies (2) in $\S 4$. This proof involves topology and certain deformation theory arguments.

In $\S 5$, we describe classes of smooth double planes branched over curves of degree $2 s$ for every $s \geq 1$ which admit Ulrich line bundles, and give examples. When $X$ is branched over a conic $B$, then Ulrich line bundles arise from lines in $\mathbb{P}^{2}$ which are simple tangents to $B$ at any point of $B$, as shown in

Corollary 1.2. Let $X$ be a smooth surface which is a double cover of $\mathbb{P}^{2}$ branched along a smooth conic curve $B$. Then $X$ admits a pair of Ulrich line bundles.

In fact, any double plane $X$ branched over a smooth conic is isomorphic to $\mathbb{P}^{1} \times \mathbb{P}^{1}$, and the two Ulrich line bundles correspond to the two rulings of $\mathbb{P}^{1} \times \mathbb{P}^{1}$, cf. $\oint 5.1$. Next, we consider double planes $X$ branched over a smooth quartic in $\S 5.2$ where we prove the following

Corollary 1.3. Let $X$ be a smooth surface which is a double cover of $\mathbb{P}^{2}$ branched along a smooth quartic curve $B$. Then $X$ admits 63 pairs of Ulrich line bundles.

Hadan [12] proves that - to any smooth plane quartic curve, there are 63 disjoint one parameter families of smooth plane conics (simple) tangentially meeting the quartic at 4 points. Using this, we obtain that a general conic in each such family gives rise to a pair of Ulrich line bundles. When $X$ is branched over a smooth degree $2 s$ curve for $s \geq 3$, we prove the theorem stated below.

Theorem 1.4. Let $X$ be a smooth surface which is a double cover of $\mathbb{P}^{2}$ branched along a generic smooth curve $B \subset \mathbb{P}^{2}$ of degree $2 s$ for $s \geq 3$. Then $X$ does not admit Ulrich line bundles.

We show that a necessary condition for any double plane $X$ to carry an Ulrich line bundle is that the Picard number $\rho(X)>1$, cf. Lemma 3.2. By a version of the NoetherLefschetz theorem, it is known that the Picard group of a double plane branched over a generic smooth degree $2 s$ curve for $s \geq 3$ is $\mathbb{Z}$ ([7], [11, Chapter 2]), which proves the theorem, cf. $\oint \underline{5.3}$.

However, for each $s \geq 3$, there are special classes of double planes which do admit Ulrich line bundles. In particular we prove the following in $\S 5.3$.

Theorem 1.5. Let $C \subset \mathbb{P}^{2}$ be a smooth degree $s$ curve. Consider an effective divisor $\mathfrak{d}$ of degree $s^{2}$ on $C$ corresponding to a section of $H^{0}\left(C, \mathcal{O}_{C}(s) \otimes L_{0}\right)$, where $L_{0} \in \operatorname{Pic} C$ is of order two.

(a) Then, there exist smooth curves $B$ of degree $2 s$ such that $B \cdot C=2 \mathfrak{d}$.

(b) Further, let $X$ be a double cover of $\mathbb{P}^{2}$ branched along a smooth curve $B$ as in part (a) of this theorem. Then, $X$ admits Ulrich line bundles.

Next, we concretely exhibit double planes branched over degree $2 s$ curves with $s$ even, which admit Ulrich line bundles.

Theorem 1.6. Let $B \subset \mathbb{P}^{2}$ be a Fermat curve of degree $2 s$ where $s$ is an even integer. Then the double plane $X$ branched along $B$ admits Ulrich line bundles. 
We show this by identifying explicitly a degree $s$ curve $C$ tangential to $B$ of even order at all points of $C \cap B$. We finally end with some remarks about the existence of Ulrich line bundles in higher dimensions. In this direction, we prove the following theorem in $\S 6$.

Theorem 1.7. Consider a smooth d-fold cyclic cover $\pi: X \rightarrow \mathbb{P}^{n}$ branched over a smooth hypersurface $B \subset \mathbb{P}^{n}$ where $n \geq 3$. If $d \leq n$, then $X$ does not admit Ulrich line bundles with respect to $\pi$.

The above theorem shows that, in general, we cannot expect Ulrich line bundles on $d$-fold cyclic coverings of $\mathbb{P}^{n}$ when $d \leq n$. Double planes, i.e. the case $d=n=2$, are the only exceptions and are indeed special in this respect.

\section{Preliminaries}

We now briefly recall some preliminaries about Ulrich bundles. Refer [6] for a comprehensive introduction to Ulrich bundles.

2.1. Ulrich bundles. The definition of Ulrich bundles which we will use in this article is as follows.

Definition 2.1. Consider a smooth projective variety $X$ admitting a finite morphism $\pi: X \rightarrow \mathbb{P}^{\operatorname{dim} X}$. A vector bundle $E$ on $X$ is said to be Ulrich with respect to the morphism $\pi$ if the direct image $\pi_{*} E$ is the trivial vector bundle.

The above definition is a special version of the general definition of Ulrich bundles, which we recall now.

Definition 2.2. Let $X$ be a smooth projective variety over $\mathbb{C}$, and $E$ be a vector bundle on $X$. Consider a very ample line bundle $\mathcal{O}_{X}(1)$ on $X$ inducing the embedding $X \hookrightarrow \mathbb{P}^{N}$. Then $E$ is Ulrich on $\left(X, \mathcal{O}_{X}(1)\right)$ if it satisfies one of the following equivalent conditions.

(1) The direct image vector bundle $\pi_{*} E$ is trivial for any finite linear projection $\pi$ :

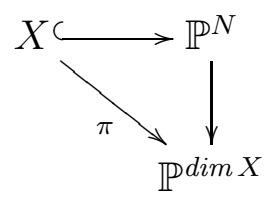

(2) $H^{i}(X, E(-p))=0$ for $1 \leq p \leq \operatorname{dim} X$ and for all $i$.

Note that, one can also define Ulrich bundles with respect to ample and globally generated polarizations as in the paper [3]. In [3, Proposition 3], the authors record that the existence of Ulrich bundles with respect to an ample and globally generated line bundle $\mathcal{O}_{X}(1)$ guarantees the existence of Ulrich bundles with respect to $\mathcal{O}_{X}(d)$ for $d>0$. Our Definition 2.1 is in fact with respect to the ample and globally generated line bundle $\mathcal{O}_{X}(1)=\pi^{*} \mathcal{O}_{\mathbb{P d i m} X}(1)$.

It is also interesting to note that Ulrich bundles have several favourable properties. For example, they are globally generated, semistable and, if not stable, they are extensions of Ulrich bundles of smaller rank. 
2.2. Double planes. We now recall some basic facts about double planes.

Consider a smooth curve $B \subset \mathbb{P}^{2}$ of even degree $2 s$ which is defined by a polynomial (section) $F \in H^{0}\left(\mathbb{P}^{2}, \mathcal{O}_{\mathbb{P}^{2}}(2 s)\right)$. We can construct a double cover $X$ of $\mathbb{P}^{2}$ branched along $B$ as follows. Let $\mathbb{A}$ denote the total space of the line bundle $A=\mathcal{O}_{\mathbb{P}^{2}}(s), p: \mathbb{A} \rightarrow \mathbb{P}^{2}$ the projection and $t \in H^{0}\left(\mathbb{A}, p^{*} A\right)$ be the tautological section. Define $X$ to be the subvariety of $\mathbb{A}$ defined by the section $t^{2}-p^{*} F \in H^{0}\left(\mathbb{A}, p^{*} A^{\otimes 2}\right)=H^{0}\left(\mathbb{A}, p^{*} \mathcal{O}_{\mathbb{P}^{2}}(2 s)\right)$. The morphism $\left.p\right|_{X}=: \pi: X \rightarrow \mathbb{P}^{2}$ is of degree two and branched over the curve $B$. We make note of the following observations.

(1) The double plane $X$ is smooth if and only if $B$ is smooth, cf. [11, Section on Double covers, Chapter II] and [17, Proposition 4.1.6].

(2) The inverse image $R \subset X$ of the branch curve $B$ is isomorphic to $B$, and $\pi^{*} A=$ $\pi^{*} \mathcal{O}_{\mathbb{P}^{2}}(s)=\mathcal{O}_{X}(R)$.

(3) The direct image $\pi_{*} \mathcal{O}_{X}$ is a rank two vector bundle on $\mathbb{P}^{2}$ since $\pi$ is a finite flat morphism. The trace map $\operatorname{Tr}: \pi_{*} \mathcal{O}_{X} \rightarrow \mathcal{O}_{\mathbb{P}^{2}}$ gives a splitting of the natural morphism $\mathcal{O}_{\mathbb{P}^{2}} \rightarrow \pi_{*} \mathcal{O}_{X}$. In particular it can be shown that $\pi_{*} \mathcal{O}_{X}=\mathcal{O}_{\mathbb{P}^{2}} \oplus$ $\mathcal{O}_{\mathbb{P}^{2}}(-s)$, cf. [17, Remark 4.1.7].

(4) The canonical bundles of $X$ and $\mathbb{P}^{2}$ are related by the following relation ( cf. [9, $\S 1.41])$ :

$$
K_{X}=\pi^{*} K_{\mathbb{P}^{2}} \otimes \mathcal{O}_{X}(R)=\pi^{*} \mathcal{O}_{\mathbb{P}^{2}}(-3+s) .
$$

\subsection{Notation and Conventions.}

(1) By an Ulrich line bundle on the double plane $\pi: X \rightarrow \mathbb{P}^{2}$, we mean an Ulrich line bundle with respect to $\pi$, i.e. a line bundle $L$ on $X$ such that $\pi_{*} L=\mathcal{O}_{\mathbb{P}^{2}} \oplus \mathcal{O}_{\mathbb{P}^{2}}$.

(2) If $\mathfrak{d}$ is an effective divisor on a curve $C$, then Supp $\mathfrak{d}$ denotes the support of $\mathfrak{d}$.

(3) If $L$ is a line bundle on a surface $X$, then $|L|_{s m}$ is the set of smooth curves in the linear system $|L|$.

(4) Tangent curves - Let $B$ and $C$ be two plane curves and $P$ be a smooth point of $B$ and $C$. Let $(B \cdot C)_{P}$ denote the intersection multiplicity of $B$ and $C$ at $P$.

- If $(B \cdot C)_{P}=1$, then $B$ and $C$ intersect transversally at the point $P$.

- If $(B \cdot C)_{P}=2$, then $B$ is called a simple tangent to $C$ at $P$ or a tangent of order 2 to $C$ at $P$.

- If $(B \cdot C)_{P}=r>2$, then $B$ is said to be tangential of order $r$ to $C$ at $P$.

\section{Conditions for the EXistence of Ulrich Line BUndles}

In this section, we discuss the conditions for the existence of Ulrich line bundles on double planes. We start by making a remark regarding the global generation of Ulrich bundles. Recall that $X$ is a double cover of $\mathbb{P}^{2}$ branched along a smooth curve $B$ of degree $2 s$.

Remark 3.1. Suppose that $L$ is an Ulrich line bundle on $X$ with respect to $\pi$. Then $\pi_{*} L \simeq \mathcal{O}_{\mathbb{P}^{2}} \oplus \mathcal{O}_{\mathbb{P}^{2}}$ and so $h^{0}(X, L)=2$. Note that in general an Ulrich bundle is always globally generated, cf. [6. Theorem 1]. When $X$ is a double plane, there is a surjective morphism of locally free sheaves ([11, Chapter 2, Lemma 29])

$$
\mathcal{O}_{X} \oplus \mathcal{O}_{X} \simeq \pi^{*} \pi_{*} L \rightarrow L \rightarrow 0
$$

which also shows the global generation of $L$ in our context. 
The following lemma gives a necessary condition for a double plane to admit Ulrich line bundles.

Lemma 3.2. Let $X$ be a smooth double plane that admits an Ulrich line bundle. Then the Picard number $\rho(X)>1$ and in particular $\operatorname{Pic} X \not \mathbb{Z}$.

Proof. Let $L$ be an Ulrich line bundle on $X$. Since $h^{0}(X, L)=2, L$ is a line bundle corresponding to the class of a non-zero effective divisor. If $\operatorname{Pic} X \simeq \mathbb{Z}$, then $L$ has to be ample. By Remark 3.1, $L$ is globally generated and we have a surjection

$$
\mathcal{O}_{X} \oplus \mathcal{O}_{X} \rightarrow L \rightarrow 0 \text {. }
$$

So $L$ is generated by two sections which correspond to curves which do not intersect in the surface $X$. Thus $(L)^{2}=0$, implying that $L$ is not ample. Hence $\rho(X)>1$.

We now state Theorem 1.1 from the Introduction and give a proof.

Theorem 1.1. Suppose that $\pi: X \rightarrow \mathbb{P}^{2}$ is a double plane branched over a smooth curve $B$ of degree $2 s$. Let $R$ denote the smooth ramified curve in $X$ and $\sigma$ denote the involution of $X$ obtained by interchanging the two sheets of the double cover. Then, the following are equivalent.

(1) The surface $X$ admits an Ulrich line bundle with respect to $\pi$.

(2) There is a smooth curve $D \subset X$ such that $D \neq \sigma(D)$ and $D \cdot \sigma(D)=D \cdot R=$ $\sigma(D) \cdot R=s^{2}$.

(3) There is a smooth curve $C$ of degree $s$ in $\mathbb{P}^{2}$ which is a tangent to $B$ of even order at every point of $C \cap B$.

Proof. As $X$ is ramified over a smooth curve $B$ of degree $2 s$, we have $\pi_{*} \mathcal{O}_{X}=\mathcal{O}_{\mathbb{P}^{2}} \oplus$ $\mathcal{O}_{\mathbb{P}^{2}}(-s)$.

Part $1-(2) \Longrightarrow(3)$. Consider a smooth curve $D \subset X$ as in the part (2) of the statement of the theorem. Let $D \cap \sigma(D)=\mathfrak{d}$ where $\mathfrak{d}$ is an effective divisor on $D$ of degree $s^{2}$. Denote $C:=\pi(D)$. Since $D \neq \sigma(D)$, we have $\pi^{-1}(C)=D+\sigma(D)$. The restricted morphism $\left.\pi\right|_{D+\sigma(D)}: D+\sigma(D) \rightarrow C$ is of degree two and in particular, it is a two sheeted covering map away from the support of $\mathfrak{d}$. In fact, the restricted map $D \rightarrow C$ is of degree one and hence is a normalization map. Thereby, we have the following relation between the arithmetic genera of $C$ and $D$ :

$$
p_{a}(C)=p_{a}(D)+\Sigma_{p \in C} \delta_{p},
$$

where $p_{a}$ denotes the arithmetic genus of the curve, and $\delta_{p}=\operatorname{length}\left(\widetilde{\mathcal{O}_{p}} / \mathcal{O}_{p}\right)$, cf. [13, Exercise IV.1.8].

Since the morphism $\pi$ is of degree two, $\pi^{*} C \cdot \pi^{*} B=2(C \cdot B)$. But

$$
\pi^{*} C \cdot \pi^{*} B=(D+\sigma(D)) \cdot 2 R .
$$

The hypothesis that $D \cdot R=\sigma(D) \cdot R=s^{2}$ gives

$$
(C \cdot B)=(D+\sigma(D)) \cdot R=2 s^{2} .
$$

Since $B$ is a degree $2 s$ curve, the Bezout's theorem [13, Corollary I.7.8] shows that $C \in$ $\left|\mathcal{O}_{\mathbb{P}^{2}}(s)\right|$, and so

$$
p_{a}(C)=\frac{(s-1)(s-2)}{2} .
$$


Next, from the adjunction formula for $D \subset X$, we get

$$
\begin{aligned}
& \omega_{D}=\left.\left(K_{X} \otimes \mathcal{O}_{X}(D)\right)\right|_{D}, \text { i.e. } \\
& 2 p_{a}(D)-2=K_{X} \cdot D+D^{2} .
\end{aligned}
$$

From $\S 2.2$ (4), the canonical line bundle of $X$ is $K_{X}=\pi^{*} \mathcal{O}_{\mathbb{P}^{2}}(s-3)$. Then,

$$
K_{X} \cdot(D+\sigma(D))=\pi^{*} \mathcal{O}_{\mathbb{P}^{2}}(s-3) \cdot \pi^{*} C=\pi^{*} \mathcal{O}_{\mathbb{P}^{2}}(s-3) \cdot \pi^{*} \mathcal{O}_{\mathbb{P}^{2}}(s)=2 s(s-3) .
$$

The curves $D$ and $\sigma(D)$ are isomorphic by a global automorphism and hence

$$
K_{X} \cdot D=s(s-3) .
$$

In order to compute $(D)^{2}$, we consider $\left(\pi^{*} C\right)^{2}=2(C)^{2}$, giving

$$
\begin{gathered}
(D+\sigma(D))^{2}=2 s^{2} \text {, i.e. } \\
D^{2}+\sigma(D)^{2}+2 D \cdot \sigma(D)=2 s^{2} .
\end{gathered}
$$

By hypothesis, $D \cdot \sigma(D)=s^{2}$ and $D^{2}=\sigma(D)^{2}$ by the symmetry of $D$ and $\sigma(D)$. Thus $D^{2}=\sigma(D)^{2}=0$. We thus compute the arithmetic genus of $D$ from (3) to be

$$
p_{a}(D)=\frac{(s-1)(s-2)}{2} .
$$

Thus $p_{a}(C)=p_{a}(D)$, and from (1) , we get $\Sigma_{p \in C} \delta_{p}=0$. Hence $C$ is a smooth plane curve, and in fact $C \simeq D \simeq \sigma(D)$. Finally, from equation (2), we get the following intersection multiplicity for any $x \in \operatorname{supp} \mathfrak{d}$,

$$
(C \cdot B)_{\pi(x)}=(D \cdot R)_{x}+(\sigma(D) \cdot R)_{x}=2(D \cdot R)_{x} .
$$

This shows that $C$ is a tangent of an even order to the branch curve $B$ at every point where it intersects B. Thus (2) implies (3).

Part 2 - (2) $\Longrightarrow(1)$. From Part 1 , we have $C:=\pi(D)$ is a smooth curve in $\mathbb{P}^{2}$ with degree $C=s$ and $D^{2}=0$. Consider the commutative diagram:

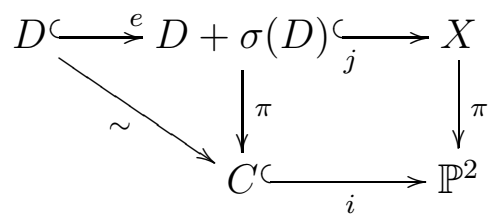

The canonical line bundle of the smooth curve $C$ is given by the adjunction formula,

$$
\omega_{C}=\left.\left(K_{\mathbb{P}^{2}} \otimes \mathcal{O}_{\mathbb{P}^{2}}(C)\right)\right|_{C}=i^{*} \mathcal{O}_{\mathbb{P}^{2}}(s-3) .
$$

Since $\pi \circ e: D \rightarrow C$ is an isomorphism,

$$
\omega_{D} \simeq e^{*} \pi^{*} i^{*} \mathcal{O}_{\mathbb{P}^{2}}(s-3) .
$$

Again, from the adjunction formula $\omega_{D}=\left.\left(K_{X} \otimes \mathcal{O}_{X}(D)\right)\right|_{D}$ for $D \subset X$, we get,

$$
\omega_{D}=\left.e^{*} j^{*} \pi^{*} \mathcal{O}_{\mathbb{P}^{2}}(s-3) \otimes \mathcal{O}_{X}(D)\right|_{D}=\left.e^{*} \pi^{*} i^{*} \mathcal{O}_{\mathbb{P}^{2}}(s-3) \otimes \mathcal{O}_{X}(D)\right|_{D} .
$$

Thus, by comparing equations (4) and (5), we get

$$
\left.\mathcal{O}_{X}(D)\right|_{D}=\mathcal{O}_{D}
$$

Consider the following short exact sequence in $X$ :

$$
0 \rightarrow \mathcal{O}_{X} \rightarrow \mathcal{O}_{X}(D) \rightarrow j_{*} e_{*} \mathcal{O}_{D} \rightarrow 0,
$$


and the associated long exact sequence of cohomology:

$$
0 \rightarrow H^{0}\left(X, \mathcal{O}_{X}\right) \rightarrow H^{0}\left(X, \mathcal{O}_{X}(D)\right) \rightarrow H^{0}\left(D, \mathcal{O}_{D}\right) \rightarrow H^{1}\left(X, \mathcal{O}_{X}\right) \rightarrow \cdots
$$

Since $\pi$ is a finite morphism, $H^{1}\left(X, \mathcal{O}_{X}\right) \simeq H^{1}\left(\mathbb{P}^{2}, \mathcal{O}_{\mathbb{P}^{2}} \oplus \mathcal{O}_{\mathbb{P}^{2}}(-s)\right)=0$. Hence,

$$
h^{0}\left(X, \mathcal{O}_{X}(D)\right)=2=h^{0}\left(\mathbb{P}^{2}, \pi_{*} \mathcal{O}_{X}(D)\right) .
$$

We next pushforward (6) to $\mathbb{P}^{2}$ by $\pi$ to get:

$$
0 \rightarrow \pi_{*} \mathcal{O}_{X} \rightarrow \pi_{*} \mathcal{O}_{X}(D) \rightarrow \pi_{*} j_{*} e_{*} \mathcal{O}_{D} \rightarrow 0
$$

Since $\pi \circ e: D \rightarrow C$ is an isomorphism, $\pi_{*} j_{*} e_{*} \mathcal{O}_{D} \simeq i_{*} \mathcal{O}_{C}$. So $c_{1}\left(\pi_{*} j_{*} e_{*} \mathcal{O}_{D}\right)=[C]=[s]$. Also, as $\pi_{*} \mathcal{O}_{X}=\mathcal{O}_{\mathbb{P}^{2}} \oplus \mathcal{O}_{\mathbb{P}^{2}}(-s)$, we have $c_{1}\left(\pi_{*} \mathcal{O}_{X}\right)=[-s]$. Hence, $c_{1}\left(\pi_{*} \mathcal{O}_{X}(D)\right)=$ $\mathcal{O}_{X}$. The two dimensional space of global sections of $\pi_{*} \mathcal{O}_{X}(D)$ gives the following exact sequence with cokernel $T$ :

$$
0 \rightarrow \mathcal{O}_{\mathbb{P}^{2}} \oplus \mathcal{O}_{\mathbb{P}^{2}} \rightarrow \pi_{*} \mathcal{O}_{X}(D) \rightarrow T \rightarrow 0
$$

Now $T$ is a torsion sheaf with $c_{1}(T)=0$. Hence, $\operatorname{supp} T$ is a closed subset of $\mathbb{P}^{2}$ with codimension at least two in $\mathbb{P}^{2}$. That is, we have two locally free sheaves $\mathcal{O}_{\mathbb{P}^{2}} \oplus \mathcal{O}_{\mathbb{P}^{2}}$ and $\pi_{*} \mathcal{O}_{X}(D)$ which are isomorphic on an open subset of $\mathbb{P}^{2}$ whose complement has codimension $\geq 2$. Thus, by [14, Proposition 1.6 (iii)] $\pi_{*} \mathcal{O}_{X}(D) \simeq \mathcal{O}_{\mathbb{P}^{2}} \oplus \mathcal{O}_{\mathbb{P}^{2}}$. This shows that $\mathcal{O}_{X}(D)$ is an Ulrich bundle for $\pi$. Hence (2) implies (1).

Part 3 - (1) $\Longrightarrow(2)$. Assume (1), i.e. let $L$ be an Ulrich line bundle on $X$. Then, by Lemma 3.2, $(L)^{2}=0$ and by Remark 3.1, $L$ is globally generated. By Bertini's theorem [13, Corollary II.10.9], we can assume that $L=\mathcal{O}_{X}(D)$ for a smooth curve $D \stackrel{j}{\hookrightarrow} X$. Consider the short exact sequence

$$
\left.0 \rightarrow \mathcal{O}_{X} \rightarrow \mathcal{O}_{X}(D) \rightarrow j_{*} \mathcal{O}_{X}(D)\right|_{D} \rightarrow 0
$$

and push-forward to $\mathbb{P}^{2}$ by $\pi$ to get

$$
\left.0 \rightarrow \mathcal{O}_{\mathbb{P}^{2}} \oplus \mathcal{O}_{\mathbb{P}^{2}}(-s) \rightarrow \mathcal{O}_{\mathbb{P}^{2}} \oplus \mathcal{O}_{\mathbb{P}^{2}} \rightarrow \pi_{*} j_{*} \mathcal{O}_{X}(D)\right|_{D} \rightarrow 0
$$

Considering the long exact sequence of cohomology associated to the above short exact sequence, we get the following.

(a) $h^{0}\left(D,\left.\mathcal{O}_{X}(D)\right|_{D}\right)=1$. Combining with the fact that

$$
\left.\operatorname{deg} \mathcal{O}_{X}(D)\right|_{D}=(D)^{2}=(L)^{2}=0
$$

we get $\left.\mathcal{O}_{X}(D)\right|_{D}=\mathcal{O}_{D}$.

(b) Next $H^{1}\left(D,\left.\mathcal{O}_{X}(D)\right|_{D}\right) \simeq H^{2}\left(\mathbb{P}^{2}, \mathcal{O}_{\mathbb{P}^{2}}(-s)\right)$. By part (a) and Serre duality, we get $H^{1}\left(D, \mathcal{O}_{D}\right) \simeq H^{0}\left(\mathbb{P}^{2}, \mathcal{O}_{\mathbb{P}^{2}}(s-3)\right)^{\vee}$. Thereby, the genus of $D$ is $g(D)=$ $h^{0}\left(\mathbb{P}^{2}, \mathcal{O}_{\mathbb{P}^{2}}(s-3)\right)=\frac{(s-1)(s-2)}{2}$.

Now if $\sigma(D)=D$, then consider $C=D /\langle\sigma\rangle$, the quotient of $D$ by the action of $\sigma$. We then have $\pi^{*} \mathcal{O}_{\mathbb{P}^{2}}(C)=\mathcal{O}_{X}(D)$ and by projection formula,

$$
\pi_{*} \mathcal{O}_{X}(D)=\pi_{*} \pi^{*} \mathcal{O}_{\mathbb{P}^{2}}(C)=\left(\mathcal{O}_{\mathbb{P}^{2}} \oplus \mathcal{O}_{\mathbb{P}^{2}}(-s)\right) \otimes \mathcal{O}_{\mathbb{P}^{2}}(C)
$$

which is not trivial. This contradicts the fact that $\mathcal{O}_{X}(D)$ is an Ulrich bundle.

Thus $\sigma(D) \neq D$. Setting $C:=\pi(D)$, we get $\pi^{-1}(C)=D+\sigma(D)$. Let $m \in \mathbb{Z}^{+}$be such that $C \in\left|\mathcal{O}_{\mathbb{P}^{2}}(m)\right|$. Then $D+\sigma(D) \in\left|\pi^{*} \mathcal{O}_{\mathbb{P}^{2}}(m)\right|$, and hence

$$
K_{X} \cdot(D+\sigma(D))=\pi^{*} \mathcal{O}_{\mathbb{P}^{2}}(s-3) \cdot \pi^{*} \mathcal{O}_{\mathbb{P}^{2}}(m)=2 m(s-3) \text { giving }
$$




$$
K_{X} \cdot D=m(s-3) .
$$

The adjunction formula for $D \subset X$, i.e. $2 g(D)-2=K_{X} \cdot D+D^{2}$ shows that

$$
s(s-3)=m(s-3) \text { implying that } m=s .
$$

Hence $C$ is a plane curve of degree $s$ and $(D+\sigma(D))^{2}=2(C)^{2}=2 s^{2}$. That is,

$$
(D)^{2}+(\sigma(D))^{2}+2 D \cdot \sigma(D)=2 s^{2} .
$$

Note that $(D)^{2}=(\sigma(D))^{2}=0$ since $D$ and $\sigma(D)$ are isomorphic curves in $X$. Thus $D \cdot \sigma(D)=s^{2}$. Further,

$$
2\left(2 s^{2}\right)=2(C \cdot B)=\pi^{*} C \cdot \pi^{*} B=(D+\sigma(D)) \cdot 2 R .
$$

Again by the symmetry of $D$ and $\sigma(D)$, we obtain $D \cdot R=\sigma(D) \cdot R=s^{2}$. This shows that (1) $\Longrightarrow$ (2). We show that (3) $\Longrightarrow(2)$ in the next section.

As a consequence of the theorem, the following remark shows that Ulrich line bundles appear in pairs.

Remark 3.3. Let $\mathcal{O}_{X}(D)$ be an Ulrich line bundle on $X$, where $D \subset X$ is a smooth curve. Then $\sigma(D) \subset X$ is another smooth curve satisfying condition two of Theorem 1.1. Note that $\mathcal{O}_{X}(\sigma(D)) \neq \mathcal{O}_{X}(D)$. For, if $\mathcal{O}_{X}(\sigma(D))=\mathcal{O}_{X}(D)$, then $\mathcal{O}_{X}(\sigma(D)) \cdot \mathcal{O}_{X}(D)=0$. But, we know that $\mathcal{O}_{X}(\sigma(D)) \cdot \mathcal{O}_{X}(D)=\sigma(D) \cdot D=s^{2}$. Hence, $\mathcal{O}_{X}(\sigma(D))$ is another Ulrich line bundle on $X$.

The following remark discusses the morphism from $X$ to the projective line induced by an Ulrich line bundle and its fibres.

Remark 3.4. Consider an Ulrich line bundle $L$ on a double plane $X$ branched over a smooth degree $2 s$ curve $B$. Since $L$ is globally generated and $h^{0}(X, L)=2$, L gives a morphism

$$
\phi: X \rightarrow \mathbb{P}^{1} .
$$

Note that the fibres of $\phi$ are curves $D \subset X$ such that $\mathcal{O}_{X}(D)=L$. Hence, there is a one parameter family of curves in $X$ represented by the linear equivalence class $[D]$, whose general element satisfies condition (2) of Theorem 1.1. Thereby, we also have a one parameter family of curves in $\mathbb{P}^{2}$ satisfying condition (3) of the theorem, which is the image of the one parameter family of curves $[D]$ in $X$.

\section{Even ORDER TANGEnt CURVES TO THE BRANCH CURVE}

Our objective in this section is to prove that the condition (3) of Theorem 1.1 implies condition (2). As earlier, suppose that $\pi: X \rightarrow \mathbb{P}^{2}$ is a degree two morphism of smooth surfaces. Let $B \in\left|\mathcal{O}_{\mathbb{P}^{2}}(2 s)\right|_{\text {sm }}$ be the smooth even degree branch curve of $\pi$. Let $C \subset \mathbb{P}^{2}$ be a curve satisfying the hypotheses of part (3) of the theorem. That is, $C$ is a smooth degree $s$ curve in $\mathbb{P}^{2}$ which is a tangent to $B$ of even order at every point of $C \cap B$. Let $B \cap C=2 \mathfrak{d}$, where $\mathfrak{d}$ is an effective divisor of degree $s^{2}$ on $B$ (therefore on $C$ as well) such that $\operatorname{Supp} \mathfrak{d}=\left\{P_{1}, P_{2}, \cdots, P_{r}\right\}$. Let us also use the same notation to denote the preimages of these points in $X$. We now prove the local reducibility of $\pi^{-1}(C)$.

Lemma 4.1. The inverse image curve $\pi^{-1}(C)$ is locally reducible at each of the points $P_{1}, P_{2}, \cdots, P_{r}$ in $X$. 
Proof. Consider a local chart in $\mathbb{P}^{2}$ around $P_{i}$ which maps $P_{i}$ to the origin $(0,0) \in \mathbb{C}^{2}$. Since both $C$ and $B$ in this affine patch pass through $(0,0)$, their local equations in the complete local ring $\mathbb{C}[[x, y]]$ contain no constant terms. By a local change of coordinates, set the local equation of $C$ as $x^{\prime}=0$. Let $l \in \mathbb{N}$ be such that $(B \cdot C)_{P_{i}}=2 l$, i.e. $B$ and $C$ are tangent of order $2 l$ to each other at $P_{i}$. Then in the coordinates $x^{\prime}, y$, the local equation of $B$ in $\mathbb{C}\left[\left[x^{\prime}, y\right]\right]$ can be written as

$$
x^{\prime}(a+f)+y^{2 l}(b+g)=0,
$$

where $a, b \in \mathbb{C}^{*}$ and $f, g \in\left(x^{\prime}, y\right)$. Thus the local equation of $B$ is

$$
u_{1} x^{\prime}+u_{2} y^{2 l}=0
$$

where we note that $u_{1}=(a+f), u_{2}=(b+g)$ are units in $\mathbb{C}\left[\left[x^{\prime}, y\right]\right]$. Since $\frac{u_{2}}{u_{1}}$ and $\left(\frac{u_{2}}{u_{1}}\right)^{\frac{1}{2 l}}$ are units in $\mathbb{C}\left[\left[x^{\prime}, y\right]\right]$, we change the local coordinates to make the local equation of $B$

$$
x^{\prime}+\left(y^{\prime}\right)^{2 l}=0 .
$$

Finally set $x_{1}=x^{\prime}+\left(y^{\prime}\right)^{2 l}$. Then, in the local coordinates $x_{1}, y^{\prime}$, the local equation of $B$ is $\left(x_{1}=0\right)$ and the local equation of $C$ is $\left(x_{1}-\left(y^{\prime}\right)^{2 l}=0\right)$.

Let $u, v$ be the local coordinates around the point $P_{i}$ in $X$. Note that, locally around $P_{i}$, the double cover morphism $\pi: X \rightarrow \mathbb{P}^{2}$ is given by the following map of complete local rings :

$$
\mathbb{C}\left[\left[x_{1}, y^{\prime}\right]\right] \rightarrow \mathbb{C}[[u, v]] \quad x_{1} \mapsto u^{2}, y^{\prime} \mapsto v .
$$

Then the local equation $\left(x_{1}-\left(y^{\prime}\right)^{2 l}=0\right)$ of the curve $C$ in $\mathbb{P}^{2}$ pulls back to the local curve $\left(u^{2}-v^{2 l}=0\right)=\left(\left(u-v^{l}\right)\left(u+v^{l}\right)=0\right)$ in $X$, which is reducible. This shows that $\pi^{-1}(C)$ is locally reducible at $P_{i}$.

Remark 4.2. Suppose that $D_{1}$ and $D_{2}$ are curves in $X$ whose local equations in the completion $\mathbb{C}[[u, v]]$ of the stalk $\mathcal{O}_{X, P_{i}}$ at $P_{i}$ are $\left(u-v^{l}=0\right)$ and $\left(u+v^{l}=0\right)$. Then the local intersection multiplicity of $D_{1}$ and $D_{2}$ at $P_{i}$ is

$$
\left(D_{1} \cdot D_{2}\right)_{P_{i}}=\operatorname{dim}_{\mathbb{C}} \frac{\mathbb{C}[[u, v]]}{\left(u-v^{l}, u+v^{l}\right)}=\operatorname{dim}_{\mathbb{C}} \frac{\mathbb{C}[[v]]}{\left(v^{l}\right)}=l .
$$

Note that the local equation of the ramified curve $R \subset X$ in the completion $\mathbb{C}[[u, v]]$ of the stalk $\mathcal{O}_{X, P_{i}}$ at $P_{i}$ is $(u=0)$. Hence, for $j=1,2$ the local intersection multiplicity

$$
\left(D_{j} \cdot R\right)_{P_{i}}=\operatorname{dim}_{\mathbb{C}} \frac{\mathbb{C}[[u, v]]}{\left(u \pm v^{l}, u\right)}=\operatorname{dim}_{\mathbb{C}} \frac{\mathbb{C}[[v]]}{\left(v^{l}\right)}=l .
$$

Lemma 4.1 shows that, at each of the points $P_{i}$, the inverse image curve $\pi^{-1}(C)$ in fact locally splits up into two components. We next wish to show that $\pi^{-1}(C)$ is globally reducible in $X$. In order to do that, we first prove the following general lemma.

Lemma 4.3. Let $C_{1}$ and $C_{2}$ be two smooth curves in $\mathbb{P}^{2}$. Then the image of the morphism of fundamental groups:

$$
\pi_{1}\left(C_{1} \backslash C_{1} \cap C_{2}\right) \rightarrow \pi_{1}\left(\mathbb{P}^{2} \backslash C_{2}\right)
$$

is generated only by the images of the loops in $C_{1} \backslash C_{1} \cap C_{2}$ around the punctures. 
Proof. Let $C_{1} \in\left|\mathcal{O}_{\mathbb{P}^{2}}(d)\right|_{s m}$ and $C_{2} \in\left|\mathcal{O}_{\mathbb{P}^{2}}(l)\right|_{s m}$. The set

$$
\mathcal{D}=\left\{[C] \in\left|\mathcal{O}_{\mathbb{P}^{2}}(d)\right|: C \text { does not intersect } C_{2} \text { transversally }\right\}
$$

is a divisor in the projective space $\left|\mathcal{O}_{\mathbb{P}^{2}}(d)\right|$. Then the complement $\mathcal{D}^{c}$ of $\mathcal{D}$ is an open subset of $\left|\mathcal{O}_{\mathbb{P}^{2}}(d)\right|$ consisting of curves which intersect $C_{2}$ transversally.

Let $\mathcal{C}$ denote the incidence variety

$$
\mathcal{C}:=\left\{(x, C): C \in\left|\mathcal{O}_{\mathbb{P}^{2}}(d)\right|_{s m} \text { and } x \in C\right\} \subset \mathbb{P}^{2} \times\left|\mathcal{O}_{\mathbb{P}^{2}}(d)\right|_{s m},
$$

and $p: \mathcal{C} \rightarrow\left|\mathcal{O}_{\mathbb{P}^{2}}(d)\right|_{s m}$ the projection. The fibre of $p$ over a point $[C] \in\left|\mathcal{O}_{\mathbb{P}^{2}}(d)\right|_{s m}$ is $p^{-1}([C]) \simeq C$. By the Ehresmann's fibration theorem [4, Theorem 13.1.3], $p: \mathcal{C} \rightarrow$ $\left|\mathcal{O}_{\mathbb{P}^{2}}(d)\right|_{s m}$ is a locally trivial topological fibration. Consequently, by denoting the open set $p^{-1}\left(\mathcal{D}^{c} \cap\left|\mathcal{O}_{\mathbb{P}^{2}}(d)\right|_{s m}\right) \subset \mathcal{C}$ by $\mathcal{C}^{*}$, we have a relative locally trivial fibration:

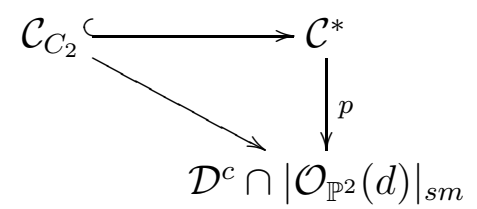

where $\mathcal{C}_{C_{2}}:=\left\{\left.\left(x, C \cap C_{2}\right)\left|C \in \mathcal{D}^{c} \cap\right| \mathcal{O}_{\mathbb{P}^{2}}(d)\right|_{s m}, x \in C \cap C_{2}\right\}$ is the relative family of divisors.

Consider a connected and smooth (it is enough to assume smoothness in $\mathcal{C}^{\infty}$ sense) curve $T \subset\left|\mathcal{O}_{\mathbb{P}^{2}}(d)\right|$ and a fixed closed point $0 \in T$ with the following property. There is a family of smooth curves $\left\{V_{t}\right\}$ of degree $d$ for $t \in T \backslash\{0\}$ which degenerates to a curve $V_{0} \in \mathcal{D}^{c}$ where $V_{0}$ is a degree $d$ curve in $\mathbb{P}^{2}$ which is a union of $d$ distinct lines $L_{1}, L_{2}, \cdots, L_{d}$ which are concurrent at a point $O \notin C_{2}$. The condition that $V_{0} \in \mathcal{D}^{c}$ implies that each $L_{i}$ intersects $C_{2}$ transversally at all points of $L_{i} \cap C_{2}$. Since $V_{0} \in \mathcal{D}^{c}$, there is a non-empty open subset of $T \backslash\{0\}$ such that the corresponding curves $V_{t} \in \mathcal{D}^{c}$.

By an infinitesimal deformation, the curve $C_{1}$ can be deformed to a curve $C_{1}^{\prime} \in \mathcal{D}^{c} \cap$ $\left|\mathcal{O}_{\mathbb{P}^{2}}(d)\right|_{s m}$. In order to prove the claim of the lemma, it is enough to prove that the image of the morphism,

$$
\pi_{1}\left(C_{1}^{\prime} \backslash C_{1}^{\prime} \cap C_{2}\right) \rightarrow \pi_{1}\left(\mathbb{P}^{2} \backslash C_{2}\right)
$$

is generated only by the images of the loops in $C_{1}^{\prime} \backslash C_{1}^{\prime} \cap C_{2}$ around the punctures.

Choose a $t_{0} \in T$ close to 0 such that $V_{t_{0}} \in \mathcal{D}^{c} \cap\left|\mathcal{O}_{\mathbb{P}^{2}}(d)\right|_{s m}$. Since $C_{1}^{\prime}, V_{t_{0}} \in \mathcal{D}^{c} \cap$ $\left|\mathcal{O}_{\mathbb{P}^{2}}(d)\right|_{s m}$, there is a path in $\mathcal{D}^{c} \cap\left|\mathcal{O}_{\mathbb{P}^{2}}(d)\right|_{s m}$ joining $C_{1}^{\prime}$ and $V_{t_{0}}$. Note that, as $\mathcal{C}_{C_{2}} \hookrightarrow \mathcal{C}^{*}$ is a relative locally trivial fibration over $\mathcal{D}^{c} \cap\left|\mathcal{O}_{\mathbb{P}^{2}}(d)\right|_{s m}, C_{1}^{\prime} \backslash\left(C_{1}^{\prime} \cap C_{2}\right)$ and $V_{t_{0}} \backslash\left(V_{t_{0}} \cap C_{2}\right)$ are homotopy equivalent. Thus, it is enough to prove that the image of

$$
\pi_{1}\left(V_{t_{0}} \backslash\left(V_{t_{0}} \cap C_{2}\right)\right) \rightarrow \pi_{1}\left(\mathbb{P}^{2} \backslash C_{2}\right)
$$

is generated only by the images of the loops in $V_{t_{0}} \backslash\left(V_{t_{0}} \cap C_{2}\right)$ around the punctures.

We do this by considering the Milnor fibration [19] around $0 \in T$. In particular, choose an $\epsilon>0$ such that the open ball $B_{\epsilon}$ and it's closure $\overline{B_{\epsilon}}$ in $\mathbb{P}^{2}$ satisfy $B_{\epsilon} \subset \overline{B_{\epsilon}} \subset \mathbb{P}^{2} \backslash C_{2}$ and $V_{t_{0}} \cap B_{\epsilon} \neq \emptyset$. If $B_{\epsilon}^{c}$ denotes the complement of the ball $B_{\epsilon}$ in $\mathbb{P}^{2}$, then the set $\left(V_{t_{0}} \backslash V_{t_{0}} \cap C_{2}\right) \cap B_{\epsilon}^{c}$ is diffeomorphic to $\bigcup_{i=1}^{d}\left(L_{i} \backslash\left(L_{i} \cap C_{2}\right)\right) \cap B_{\epsilon}^{c}$. Now consider the covering by closed sets

$$
V_{t_{0}} \backslash\left(V_{t_{0}} \cap C_{2}\right) \simeq\left(V_{t_{0}} \backslash V_{t_{0}} \cap C_{2}\right) \cap \overline{B_{\epsilon}} \cup \bigcup_{i=1}^{d}\left(L_{i} \backslash\left(L_{i} \cap C_{2}\right)\right) \cap B_{\epsilon}^{c} .
$$


Denote $A:=\left(V_{t_{0}} \backslash V_{t_{0}} \cap C_{2}\right) \cap \overline{B_{\epsilon}}$ and $A_{i}:=\left(L_{i} \backslash\left(L_{i} \cap C_{2}\right)\right) \cap B_{\epsilon}^{c}$ for $i=1,2, \cdots, d$. Each $A \cap A_{i}$ is path connected, since it is the boundary of a disc. Hence, we apply Van Kampen theorem [15, $§ 1.2]$ (using closed sets) to obtain the fundamental group $A \cup A_{1}$ at a basepoint on $A \cap A_{1}$ :

$$
\pi_{1}\left(A \cup A_{1}\right) \simeq \pi_{1}(A) *_{\pi_{1}\left(A \cap A_{1}\right)} \pi_{1}\left(A_{1}\right)
$$

Since $A \cup A_{1}$ is path-connected, the fundamental group is independent of the choice of basepoint. Next, $\left(A \cup A_{1}\right) \cap A_{2}=A \cap A_{2}$ which is again path connected. By the Van Kampen theorem, the fundamental group of $A \cup A_{1} \cup A_{2}$ based at a point of $A \cap A_{2}$ is

$$
\begin{aligned}
\pi_{1}\left(A \cup A_{1} \cup A_{2}\right) \simeq \pi_{1}\left(A \cup A_{1}\right) *_{\pi_{1}\left(A \cap A_{2}\right)} \pi_{1}\left(A_{2}\right) & \\
& \simeq \pi_{1}(A) *_{\pi_{1}\left(A \cap A_{1}\right)} \pi_{1}\left(A_{1}\right) *_{\pi_{1}\left(A \cap A_{2}\right)} \pi_{1}\left(A_{2}\right) .
\end{aligned}
$$

Inductively, we get that $\pi_{1}\left(V_{t_{0}} \backslash\left(V_{t_{0}} \cap C_{2}\right)\right)$ is isomorphic to

$$
\pi_{1}(A) *_{\pi_{1}\left(A \cap A_{1}\right)} \pi_{1}\left(A_{1}\right) *_{\pi_{1}\left(A \cap A_{2}\right)} \pi_{1}\left(A_{2}\right) \cdots *_{\pi_{1}\left(A \cap A_{d}\right)} \pi_{1}\left(A_{d}\right) .
$$

Note that by the universal property of free products, and by the definition of amalgamation products, we get the following commutative diagram.

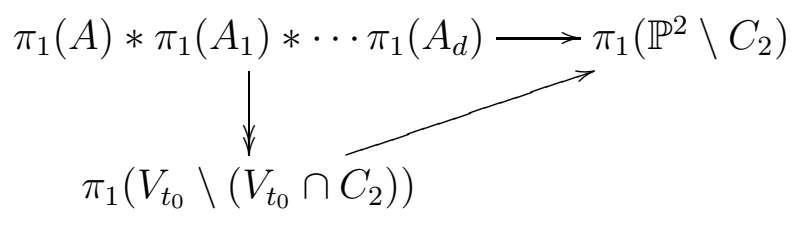

Thus it is enough to show that the image of the morphism

$$
\pi_{1}(A) * \pi_{1}\left(A_{1}\right) * \cdots \pi_{1}\left(A_{d}\right) \rightarrow \pi_{1}\left(\mathbb{P}^{2} \backslash C_{2}\right)
$$

is generated by the images of loops in $V_{t_{0}} \backslash\left(V_{t_{0}} \cap C_{2}\right)$ around the punctures. Note that, $A=\left(V_{t_{0}} \backslash V_{t_{0}} \cap C_{2}\right) \cap \overline{B_{\epsilon}} \subset \overline{B_{\epsilon}}$ is contractible in $\mathbb{P}^{2} \backslash C_{2}$. Further, each $A_{i}=\left(L_{i} \backslash\left(L_{i} \cap\right.\right.$ $\left.\left.C_{2}\right)\right) \cap B_{\epsilon}^{c}$ is homotopically equivalent to a closed disc with $l$ punctures whose fundamental group is generated by loops $\gamma_{1}, \gamma_{2}, \cdots, \gamma_{l}$ around the $l$ punctures and the boundary loop $\gamma_{1} * \gamma_{2} * \cdots \gamma_{l}$. Each of the loops $\gamma_{i}$ maps to a loop $\delta_{i}$ around $C_{2}$ in $\mathbb{P}^{2} \backslash C_{2}$, and each such $\delta_{i}$ is homotopic to the generator of $\pi_{1}\left(\mathbb{P}^{2} \backslash C_{2}\right) \simeq \mathbb{Z} / l \mathbb{Z}$. Thus the image of the boundary loop $\gamma_{1} * \gamma_{2} * \cdots * \gamma_{l}$ in $\pi_{1}\left(\mathbb{P}^{2} \backslash C_{2}\right)$ is $l \times$ (generator) which is the trivial element of $\pi_{1}\left(\mathbb{P}^{2} \backslash C_{2}\right) \simeq \mathbb{Z} / l \mathbb{Z}$.

Thereby we get that the image of

$$
\pi_{1}\left(V_{t_{0}} \backslash\left(V_{t_{0}} \cap C_{2}\right)\right) \rightarrow \pi_{1}\left(\mathbb{P}^{2} \backslash C_{2}\right)
$$

is generated only by the images of the loops in $V_{t_{0}} \backslash\left(V_{t_{0}} \cap C_{2}\right)$ around the punctures.

Using notations as earlier, we prove the following theorem.

Theorem 4.4. Let $C \subset \mathbb{P}^{2}$ be a smooth degree $s$ curve which is an even order tangent to the branch curve $B$ at every point of $C \cap B$. Then $\pi^{-1}(C)$ is a reducible curve $D_{1}+D_{2}$. Further, each component $D_{i}$ is isomorphic to $C, D_{2}=\sigma\left(D_{1}\right)$ and $D_{1} \cdot D_{2}=s^{2}=D_{i} \cdot R$, for $i=1,2$. 
Proof. Recall that $R \subset X$ denotes the ramified curve of the double cover $\pi: X \rightarrow \mathbb{P}^{2}$. Then $\pi: X \backslash R \rightarrow \mathbb{P}^{2} \backslash B$ is a genuine two sheeted covering map. Hence we have the following morphisms between the fundamental groups.

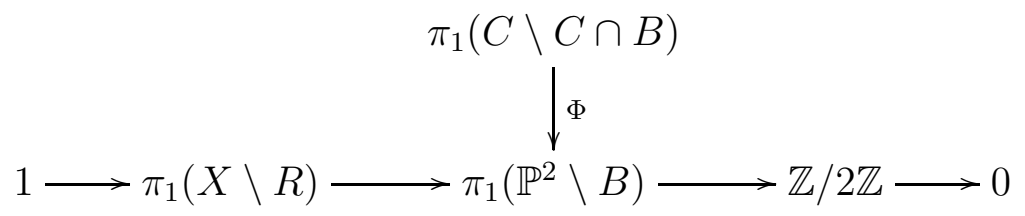

Suppose the above map of fundamental groups $\Phi: \pi_{1}(C \backslash C \cap B) \rightarrow \pi_{1}\left(\mathbb{P}^{2} \backslash B\right)$ lifts to $\pi_{1}(X \backslash R)$, then the inclusion $C \backslash C \cap B \hookrightarrow \mathbb{P}^{2} \backslash B$ lifts to $X \backslash R$. This gives a section of $\left.\pi\right|_{\pi^{-1}(C \backslash C \cap B)}: \pi^{-1}(C \backslash C \cap B) \rightarrow C \backslash C \cap B$ which shows that $\pi^{-1}(C \backslash C \cap B)$ is disconnected and thereby $\pi^{-1}(C)$ is reducible. So it is enough to prove that the morphism $\Phi$ lifts to $\pi_{1}(X \backslash R)$, which is the same as showing that the image in $\mathbb{Z} / 2 \mathbb{Z}$ of $\Phi\left(\pi_{1}(C \backslash C \cap B)\right)$ is the trivial element.

By Lemma 4.3, $\Phi\left(\pi_{1}(C \backslash C \cap B)\right)$ is generated only by loops in $C \backslash C \cap B$ around the punctures (i.e. points of $C \cap B$ ). Let $x \in C \cap B$ and let $\gamma_{x}$ be a loop in $C \backslash C \cap B$ around $x$. Then under $\Phi$,

$$
\gamma_{x} \mapsto(C \cdot B)_{x} \delta_{x}
$$

where $\delta_{x}$ is a small loop around $B$ at $x$. But such a $\delta_{x}$ can be considered a generator of $\pi_{1}\left(\mathbb{P}^{2} \backslash B\right)$ and $(C \cdot B)_{x}$ is even by hypothesis. Hence $\gamma_{x}$ maps to an even multiple of the generator of $\pi_{1}\left(\mathbb{P}^{2} \backslash B\right)$, whose further image in $\mathbb{Z} / 2 \mathbb{Z}$ is trivial. This shows that $\pi^{-1}(C)$ is reducible and has the form $D_{1}+D_{2}$.

Now $\left.\pi\right|_{D_{1}+D_{2}}: D_{1}+D_{2} \rightarrow C$ is a degree two finite map. Since $C \cap B=2 \mathfrak{d}$ where $\mathfrak{d}$ is a divisor on $B$ of degree $s^{2}$, the map $\left.\pi\right|_{D_{1}+D_{2}}$ is ramified exactly along the inverse image of $\mathfrak{d}$. Away from the ramification points $\left.\pi\right|_{D_{1}+D_{2}}$ is a covering map. Hence each $D_{i}$ is isomorphic to $C$. Thus, $D_{2}=\sigma\left(D_{1}\right)$ and $\pi^{-1}(C)=D_{1}+\sigma\left(D_{1}\right)$. From Remark 4.2, we see that $D_{1} \cdot D_{2}=\Sigma\left(D_{1} \cdot D_{2}\right)_{P_{i}}=s^{2}$ where the summation is taken over points $P_{i}$ in the inverse image of the divisor $\mathfrak{d}$. Similarly, the local intersection multiplicity computation in Remark 4.2 also shows that $D_{i} \cdot R=s^{2}$, for $i=1,2$.

Proof of Theorem 1.1 ((3) $\Longrightarrow$ (2)). Theorem 4.4 proves that the condition (3) of Theorem 1.1 implies condition (2).

\section{UlRich LINE BUNDLES ON DOUBle PLANES}

In this section, we study the existence of Ulrich line bundles over smooth double planes. We describe classes of double planes $X$ branched along a smooth degree $2 s$ curve $B$ and Ulrich line bundles over them, for every $s$. For a fixed $B \in\left|\mathcal{O}_{\mathbb{P}^{2}}(2 s)\right|_{s m}$, denote

$$
\mathfrak{T}_{B}=\left\{C \in\left|\mathcal{O}_{\mathbb{P}^{2}}(s)\right|_{s m} \mid B \cap C=2 \mathfrak{d}, \mathfrak{d} \text { an effective divisor of degree } s^{2} \text { on } B\right\} .
$$

Then $\mathfrak{T}_{B}$ consists of smooth degree $s$ curves $C$ which are even order tangents to $B$ at every point in $C \cap B$. By Theorem 1.1, the non-emptiness of $\mathfrak{T}_{B}$ implies the existence of Ulrich line bundles on $X$. Note that, by Remark 3.4, if $\mathfrak{T}_{B}$ is non-empty, then it contains at least a one parameter family of curves.

Remark 5.1. While defining the set $\mathfrak{T}_{B}$ in (77), we could assume more generally that the divisor $\mathfrak{d} \subset B$ is reduced. Indeed, consider an Ulrich line bundle $\mathcal{O}_{X}(D)$ on $X$ coming from 
$a C \in \mathfrak{T}_{B}$. This line bundle gives a morphism $\phi: X \rightarrow \mathbb{P}^{1}$, whose fibres are precisely the one parameter family of divisors in $X$ which are elements of $|D|$. Consider the restriction of $\phi$ to the ramified curve $R$ i.e. $\left.\phi\right|_{R}: R \rightarrow \mathbb{P}^{1}$, which is finite of degree $s^{2}$. By Sard's Lemma, the general fibre which is of the form $D \cap R$ of $\left.\phi\right|_{R}$ is non-singular. Hence, a general $D \cap R$ is a reduced divisor on $R$ consisting of $s^{2}$ points. Since $C \cap B=\pi(D \cap R)$, for a general $C$, the set $C \cap B$ consists of precisely $s^{2}$ points, and the corresponding divisor $\mathfrak{d}$ (whose support is $C \cap B$ ) is reduced.

5.1. The branch curve is a conic $(s=1)$. It is known that a double plane $X$ branched over a smooth conic is isomorphic to $\mathbb{P}^{1} \times \mathbb{P}^{1}$. So, Pic $X=\mathbb{Z} \oplus \mathbb{Z}$. Consider generators $l$ and $m$ of type $(0,1)$ and $(1,0)$.

Corollary 1.2. Let $X$ be a smooth surface which is a double cover of $\mathbb{P}^{2}$ branched along a smooth conic curve $B$. Then $X$ admits a pair of Ulrich line bundles.

Proof. Suppose $\pi: X \rightarrow \mathbb{P}^{2}$ is a smooth double cover branched along a conic $B \subset \mathbb{P}^{2}$. In this case

$$
\mathfrak{T}_{B}=\left\{C \in\left|\mathcal{O}_{\mathbb{P}^{2}}(1)\right|_{\text {sm }} \mid B \cap C=2 \mathfrak{d}, \mathfrak{d} \text { an effective divisor of degree } 1 \text { on } B\right\} .
$$

That is, $\mathfrak{T}_{B}$ consists of lines $C \subset \mathbb{P}^{2}$ which are simple tangents to the conic $B$ at any point $P \in B$. This set $\mathfrak{T}_{B}$ is clearly non-empty. Hence, by Theorem 1.1, the double plane $X$ admits Ulrich line bundles.

For $P \in B$, let $C_{P}$ denote a line in $\mathbb{P}^{2}$ tangent to $B$ at $P$. Then the inverse image of $C_{P} \in \mathfrak{T}_{B}$, is $\pi^{-1}\left(C_{P}\right)=D_{P}+D_{P}^{\prime}$, where $D_{P}$ and $D_{P}^{\prime}$ are isomorphic to $C_{P}$ and $D_{P} \cdot D_{P}^{\prime}=1$ by Theorem 4.4. This also gives $D_{P}^{2}=D_{P}^{\prime 2}=0$. Hence $D_{P}, D_{P}^{\prime}$ correspond to divisors in the linear systems $|l|$ and $|m|$ (i.e. corresponding to the rulings $(0,1)$ and $(1,0)$ ). Hence $X$ admits precisely two Ulrich bundles given by $\mathcal{O}_{X}(l)$ and $\mathcal{O}_{X}(m)$.

5.2. The branch curve is a quartic $(s=2)$. A smooth projective surface $X$ branched along a smooth quartic curve $B$ is a Del Pezzo surface obtained by blowing up $\mathbb{P}^{2}$ at 7 points in general position. Thus $\operatorname{Pic} X \simeq \mathbb{Z}^{\oplus 8}$.

Hadan, in [12, $\S 2.1, \S 2.2]$, analyses the tangent conics to a given smooth quartic $B$. He defines a tangent conic to $B$ to be a smooth conic which intersects $B$ at 4 points each being a point of simple tangency. From the short exact sequence,

$$
0 \rightarrow \mathcal{O}_{\mathbb{P}^{2}}(-2) \rightarrow \mathcal{O}_{\mathbb{P}^{2}}(2) \rightarrow \mathcal{O}_{B}(2) \rightarrow 0,
$$

we obtain that $H^{0}\left(\mathbb{P}^{2}, \mathcal{O}_{\mathbb{P}^{2}}(2)\right) \simeq H^{0}\left(B, \mathcal{O}_{B}(2)\right)$. Hence any conic is determined by its restriction to $B$. Using this basic fact, Hadan studies conics corresponding to divisors of the form $2 \mathfrak{d}$, where $\mathcal{O}_{B}(2 \mathfrak{d})=\mathcal{O}_{B}(2)$ and $\mathfrak{d}$ is an effective divisor on $B$ of degree 4 . It is shown that to any smooth plane quartic, the variety of tangent conics is a union of 63 disjoint one parameter families, cf. [12, Proposition 2.1].

Corollary 1.3. Let $X$ be a smooth surface which is a double cover of $\mathbb{P}^{2}$ branched along a smooth quartic curve $B$. Then $X$ admits 63 pairs of Ulrich line bundles.

Proof. In this case, $\mathfrak{T}_{B}$ is given by

$$
\left\{C \in\left|\mathcal{O}_{\mathbb{P}^{2}}(2)\right|_{s m} \mid B \cap C=2 \mathfrak{d}, \mathfrak{d} \text { an effective divisor of degree } 4 \text { on } B\right\} .
$$


By the discussion above, $\mathfrak{T}_{B}$ is non-empty and in fact consists of 63 disjoint one parameter families. By Theorem 4.4, for each $C \in \mathfrak{T}_{B}$, the inverse image can be written as $\pi^{-1}(C)=$ $D+\sigma(D)$. In fact, each one parameter family of tangent conics in $\mathfrak{T}_{B}$ gives rise to two classes $[D]$ and $[\sigma(D)]$ of curves in $X$, and hence two Ulrich line bundles $\mathcal{O}_{X}(D)$ and $\mathcal{O}_{X}(\sigma(D))$ on $X$. Hence, $X$ admits 63 pairs of Ulrich line bundles.

Remark 5.2. When $s=1$ and $s=2$, we can prove that the inverse image $\pi^{-1}(C)$ for a $C \in \mathfrak{T}_{B}$ is reducible without appealing to Theorem 4.4. When $s=1,2$, from the genusdegree formula, a smooth $C \simeq \mathbb{P}^{1}$. For any $C$, consider the inverse image curve $\pi^{-1}(C)$ in $X$. The normalization $\pi^{-1}(C)$ of $\pi^{-1}(C)$ is an etale double cover of $C$. But there are no irreducible etale double covers of $\mathbb{P}^{1}$. The only etale double cover of $\mathbb{P}^{1}$ is of the form $\mathbb{P}^{1} \sqcup \mathbb{P}^{1}$. Thereby, $\widehat{\pi^{-1}(C)}$ and hence $\pi^{-1}(C)$ are both reducible. This proves that $\pi^{-1}(C)$ is of the form $D_{1}+D_{2}$ where $D_{i}$ 's are smooth curves isomorphic to $C$. However, the same argument will not be valid when $s>2$, since we will be dealing with curves of higher genus and their etale covers.

5.3. The branch curve is of degree $2 s$ when $s>2$. When $s=3$, then we can check that $K_{X} \simeq \mathcal{O}_{X}$ and $H^{1}\left(X, \mathcal{O}_{X}\right)=0$, whereby $X$ is a $K 3$ surface. For $s>3$, it is known that $X$ is a surface of general type. The proof of Theorem 1.4 is an immediate consequence of Lemma 3.2 .

Theorem 1.4. Let $X$ be a smooth surface which is a double cover of $\mathbb{P}^{2}$ branched along a generic smooth curve $B \subset \mathbb{P}^{2}$ of degree $2 s$ for $s \geq 3$. Then $X$ does not admit Ulrich line bundles.

Proof. When $X$ is a generic double plane branched over a smooth curve $B$ of degree $2 s$, with $s>2$, then Pic $X \simeq \mathbb{Z}$, cf. [11, Chapter II], [7]. Thus by Lemma 3.2, a generic double plane branched over a degree $2 s$ curve for $s>2$ does not admit Ulrich line bundles.

In particular, the above theorem implies that for a generic $B \in\left|\mathcal{O}_{\mathbb{P}^{2}}(2 s)\right|_{\text {sm }}$ with $s>2$, we have $\mathfrak{T}_{B}=\emptyset$. Thus starting from a general smooth degree $2 s$ curve $(s>2)$, one cannot find smooth degree $s$ curves everywhere tangent to it to an even order. However, we now show that starting with a smooth degree $s$ curve $C$, we can find smooth degree $2 s$ curves $B$ which are even order tangential to $C$ at all points of $C \cap B$. Thus, the double planes branched over such $B$-s admit Ulrich line bundles.

Proposition 5.3. Let $C$ be a smooth curve of degree $s$ in $\mathbb{P}^{2}$. Then there exist smooth degree $2 s$ curves $B$ such that $B \cdot C=2 \mathfrak{d}$ where $\mathfrak{d}$ is an effective divisor of degree $s^{2}$ on $C$. That is, there exist smooth curves $B$ of degree $2 s$ such that $B$ is tangential to $C$ to an even order at every point of $B \cap C$.

Proof. Suppose that $C$ is the zero set of the degree $s$ homogeneous polynomial $F(x, y, z)$ where $x, y, z$ are the homogeneous coordinates in $\mathbb{P}^{2}$. Let us denote the inclusion $C \hookrightarrow \mathbb{P}^{2}$ by $i$. Now consider the following short exact sequence:

$$
0 \rightarrow \mathcal{O}_{\mathbb{P}^{2}}(-s) \stackrel{\otimes F}{\longrightarrow} \mathcal{O}_{\mathbb{P}^{2}} \rightarrow i_{*} \mathcal{O}_{C} \rightarrow 0 .
$$

Tensoring by $\mathcal{O}_{\mathbb{P}^{2}}(2 s)$, we get

$$
0 \rightarrow \mathcal{O}_{\mathbb{P}^{2}}(s) \stackrel{\otimes F}{\longrightarrow} \mathcal{O}_{\mathbb{P}^{2}}(2 s) \rightarrow i_{*} \mathcal{O}_{C}(2 s) \rightarrow 0 .
$$


The long exact sequence of cohomology associated to the above short exact sequence gives

$$
0 \rightarrow H^{0}\left(\mathbb{P}^{2}, \mathcal{O}_{\mathbb{P}^{2}}(s)\right) \stackrel{\otimes F}{\longrightarrow} H^{0}\left(\mathbb{P}^{2}, \mathcal{O}_{\mathbb{P}^{2}}(2 s)\right) \rightarrow H^{0}\left(C, \mathcal{O}_{C}(2 s)\right) \rightarrow 0 .
$$

Therefore,

$$
H^{0}\left(C, \mathcal{O}_{C}(2 s)\right) \simeq \frac{H^{0}\left(\mathbb{P}^{2}, \mathcal{O}_{\mathbb{P}^{2}}(2 s)\right)}{F \cdot H^{0}\left(\mathbb{P}^{2}, \mathcal{O}_{\mathbb{P}^{2}}(s)\right)}
$$

If $L_{0} \in$ Pic $C$ is an element of order two, then the line bundle $\mathcal{O}_{C}(s) \otimes L_{0}$ on $C$ is of degree $s^{2}$, and the Riemann-Roch formula gives that

$$
h^{0}\left(C, \mathcal{O}_{C}(s) \otimes L_{0}\right)=\frac{s^{2}+3 s}{2} .
$$

For $\mathfrak{d} \in\left|\mathcal{O}_{C}(s) \otimes L_{0}\right|$, consider the corresponding divisor $2 \mathfrak{d} \in\left|\mathcal{O}_{C}(2 s)\right|$. This corresponds to an element $\bar{G} \in \frac{H^{0}\left(\mathbb{P}^{2}, \mathcal{O}_{\mathbb{P} 2}(2 s)\right)}{F \cdot H^{0}\left(\mathbb{P}^{2}, \mathcal{O}_{\mathbb{P} 2}(s)\right)}$ upto a non-zero scalar. Thus any element in the vector subspace

$$
V=\left\{\lambda G+F H: \lambda \in \mathbb{C}, H \in H^{0}\left(\mathbb{P}^{2}, \mathcal{O}_{\mathbb{P}^{2}}(s)\right)\right\}
$$

of $H^{0}\left(\mathbb{P}^{2}, \mathcal{O}_{\mathbb{P}^{2}}(2 s)\right)$ with $\lambda \neq 0$ maps to an element in $H^{0}\left(C, \mathcal{O}_{C}(2 s)\right)$ corresponding to $2 \mathfrak{d}$.

This means that any such degree $2 s$ curve $B$ in the linear system $\mathbb{P} V$ meets $C$ tangentially along the divisor $\mathfrak{d}$ and at each point in $\operatorname{Supp} \mathfrak{d}, B$ is an even order tangent to $C$. In order to prove our claim, we need to show the existence of smooth curves $B$.

By Bertini's theorem, a general element of $\mathbb{P} V$ is smooth away from the base points of this linear system. The base points of $\mathbb{P} V$ are precisely the points in the support of $\mathfrak{d}$. Let $P_{1}, P_{2}, \cdots, P_{r}$ be these points. As $P_{1}$ is a smooth point of $C$, the partial derivatives of $F$ cannot all vanish at $P_{1}$. Without loss of generality, assume that $F_{x}\left(P_{1}\right) \neq 0$. Consider the set of all $H \in H^{0}\left(\mathbb{P}^{2}, \mathcal{O}_{\mathbb{P}^{2}}(s)\right)$ with $(G+F H)_{x} \neq 0$, i.e.

$$
U_{1}=\left\{H \in H^{0}\left(\mathbb{P}^{2}, \mathcal{O}_{\mathbb{P}^{2}}(s)\right): H\left(P_{1}\right) \neq 0, H\left(P_{1}\right) \neq \frac{-G_{x}\left(P_{1}\right)}{F_{x}\left(P_{1}\right)}\right\} .
$$

Note that $U_{1}$ is an open dense subset of $H^{0}\left(\mathbb{P}^{2}, \mathcal{O}_{\mathbb{P}^{2}}(s)\right)$. Similarly consider open subsets $U_{i}$ corresponding to the points $P_{i}$ for $i=2,3, \cdots, r$. For a general $H \in \cap_{i=1}^{r} U_{i}$, the curve corresponding to $G+F H$ is a smooth curve of degree $2 s$ which meets $C$ (even order) tangentially at every point of $B \cap C$.

As remarked earlier, the above proposition gives rise to special double planes branched over degree $2 s$ curves $(s>2)$ that carry Ulrich line bundles. Using this we prove Theorem 1.5 .

Theorem 1.5. Let $C \subset \mathbb{P}^{2}$ be a smooth degree $s$ curve. Consider an effective divisor $\mathfrak{d}$ of degree $s^{2}$ on $C$ corresponding to a section of $H^{0}\left(C, \mathcal{O}_{C}(s) \otimes L_{0}\right)$, where $L_{0} \in \operatorname{Pic} C$ is of order two.

(a) Then, there exist smooth curves $B$ of degree $2 s$ such that $B \cdot C=2 \mathfrak{d}$.

(b) Further, let $X$ be a double cover of $\mathbb{P}^{2}$ branched along a smooth curve $B$ as in part (a) of this theorem. Then, $X$ admits Ulrich line bundles.

Proof. Proposition 5.3 proves Part (a) of the Theorem. Suppose $C$ is a smooth degree $s$ curve and $B$ is a smooth degree $2 s$ curve that is even order tangent to $C$ everywhere. Let $X$ be a double plane branched along $B$. Then, $C \in \mathfrak{T}_{B}$. By Theorem $4.4, \pi^{-1}(C)=D_{1}+D_{2}$, 
where $D_{2}=\sigma\left(D_{1}\right)$ and $D_{1} \cdot D_{2}=s^{2}=D_{i} \cdot R$. Thus by Theorem 1.1, $\mathcal{O}_{X}\left(D_{1}\right)$ and $\mathcal{O}_{X}\left(D_{2}\right)$ are Ulrich line bundles on $X$.

We now give a proof of Theorem 1.6, which explicitly gives examples of double planes $\pi: X \rightarrow \mathbb{P}^{2}$ branched over a degree $2 s$ curve, for each even $s>1$, which admit Ulrich line bundles.

Theorem 1.6. Let $B \subset \mathbb{P}^{2}$ be a Fermat curve of degree $2 s$ where $s$ is an even integer. Then the double plane $X$ branched along $B$ admits Ulrich line bundles.

Proof. Let $B \subset \mathbb{P}^{2}$ be a Fermat curve of degree $2 s$. Then there exists a choice of coordinates $x, y, z$ on $\mathbb{P}^{2}$ in which $B$ has the form

$$
\left(x^{2 s}-y^{2 s}-z^{2 s}=0\right) .
$$

Consider the double plane $\pi: X \rightarrow \mathbb{P}^{2}$ branched over this smooth curve $B$.

Let $C$ be the degree $s$ Fermat curve $\left(x^{s}-y^{s}-z^{s}=0\right)$. Denote the $s$-th roots of unity by $1, \xi, \xi^{2}, \cdots, \xi^{s-1}$. Then, for $a \neq 0$, at the $2 s$-points $(a: a: 0),(a: \xi a: 0), \cdots,\left(a: \xi^{s-1} a:\right.$ $0)$ and $(a: 0: a),(a: 0: \xi a), \cdots,\left(a: 0: \xi^{s-1} a\right)$ respectively, the lines $(x-y=0),(\xi x-y=$ $0), \cdots,\left(\xi^{s-1} x-y=0\right)$ and the lines $(x-z=0),(\xi x-z=0), \cdots,\left(\xi^{s-1} x-z=0\right)$ are :

- tangents of order $2 s$ to the curve $B$, and

- tangents of order $s$ to the curve $C$.

Hence, at each of above $2 s$ points, $B$ and $C$ are tangential of order $s$ to each other. This accounts for all the intersections between $B$ and $C$ since $B \cdot C=2 s^{2}$. Thereby the curve $C$ meets $B$ at $2 s$ points, each of which is a point of tangency of even order $s$ (since $s$ is even by hypothesis). Thus by Theorem 4.4, $\pi^{-1}(C)=D_{1}+D_{2}$ and each $\mathcal{O}_{X}\left(D_{i}\right)$ is an Ulrich bundle on $X$.

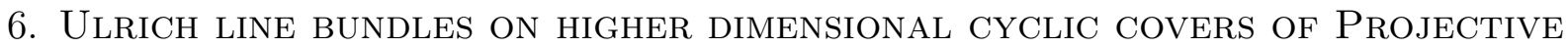 SPACE}

In this section, we make a few remarks about the existence of Ulrich line bundles on higher dimensional cyclic covers.

Theorem 1.7. Consider a smooth $d$-fold cyclic cover $\pi: X \rightarrow \mathbb{P}^{n}$ branched over a smooth hypersurface $B \subset \mathbb{P}^{n}$ where $n \geq 3$. If $d \leq n$, then $X$ does not admit Ulrich line bundles with respect to $\pi$.

Proof. Let $\pi: X \rightarrow \mathbb{P}^{n}$ be a smooth $d$-fold cyclic cover branched over a smooth hypersurface where $n \geq 3$ and $d \leq n$. If $L$ is an Ulrich line bundle on $X$, then $h^{0}(X, L)=d$ and $L$ is globally generated. That is, there is a surjective morphism

$$
\mathcal{O}_{X}^{\oplus d} \rightarrow L \rightarrow 0
$$

which shows that there are $d$ divisors of $X$ in $|L|$ which do not intersect, i.e. $L^{d}=0$.

- Double cover of $\mathbb{P}^{3}$ - If $X$ is a double cover of $\mathbb{P}^{3}$ admitting an Ulrich line bundle, then $L^{2}=0$, that is, there are two hypersurfaces $D_{1}$ and $D_{2}$ in $X$ with $D_{1} \cap D_{2}=\emptyset$. 
But this is not possible. Indeed, let $\pi\left(D_{i}\right)$ denote the hypersurfaces in $\mathbb{P}^{3}$ which are the images of $D_{i}$ for $i=1,2$. Note that

$$
\pi\left(D_{1}\right) \cap \pi\left(D_{2}\right) \cap B \neq \emptyset,
$$

since the images of $n$ hypersurfaces in $\mathbb{P}^{n}$ is not empty. This shows that $D_{1} \cap D_{2} \neq \emptyset$ and in fact they have non-empty intersection along the ramification divisor. Thus, $X$ cannot admit Ulrich line bundles.

- 3-fold cover of $\mathbb{P}^{3}$ branched along a cubic - When $X$ is a threefold cover of $\mathbb{P}^{3}$ branched along a cubic, then $X$ is a cubic hypersurface in $\mathbb{P}^{4}$. By the NoetherLefschetz theorem [17, Example 3.1.25], Pic $X=\mathbb{Z}$, and hence any non-zero effective divisor is ample. Thus $X$ cannot admit a globally generated line bundle $L$ with $L^{3}=0$. So, $X$ does not admit Ulrich line bundles.

- Other cases- The remaining cases are:

- $X$ is a threefold covering of $\mathbb{P}^{3}$ branched along a smooth hypersurface of degree $3 s$ with $s \geq 2$

- $X \rightarrow \mathbb{P}^{n}$ be a $d$-fold cover of $\mathbb{P}^{n}$ with $n \geq 4$ and $d \leq n$.

By discussion in the previous case, if $L$ is a line bundle on $X$ with $L^{d}=0$ and $h^{0}(X, L)>0$, then Pic $X \neq \mathbb{Z}$. By applications of Noether-Lefschetz theorem to the pairs $(X, R)$ and $\left(\mathbb{P}^{n}, B\right)$ and the isomorphism of $R$ and $B,[18$. prove that Pic $X=\mathbb{Z}$ in both these cases. Thus, $X$ cannot admit Ulrich line bundles.

Acknowledgements. We would like to thank N. Mohan Kumar for useful comments on the initial version of the draft. This work was supported by the Department of Atomic Energy, Government of India [project no. 12 - R\&D - TFR - 5.01 - 0500].

\section{REFERENCES}

[1] Marian Aprodu, Laura Costa, and Rosa Maria Miró-Roig. Ulrich bundles on ruled surfaces. J. Pure Appl. Algebra, 222(1):131-138, 2018.

[2] Marian Aprodu, Gavril Farkas, and Angela Ortega. Minimal resolutions, Chow forms and Ulrich bundles on K3 surfaces. J. Reine Angew. Math., 730:225-249, 2017.

[3] Marian Aprodu and Yeongrak Kim. Ulrich line bundles on Enriques surfaces with a polarization of degree four. Ann. Univ. Ferrara Sez. VII Sci. Mat., 63(1):9-23, 2017.

[4] Donu Arapura. Algebraic geometry over the complex numbers. Universitext. Springer, New York, 2012.

[5] Arnaud Beauville. Ulrich bundles on abelian surfaces. Proc. Amer. Math. Soc., 144(11):4609-4611, 2016.

[6] Arnaud Beauville. An introduction to Ulrich bundles. Eur. J. Math., 4(1):26-36, 2018.

[7] Alexandru Buium. Sur le nombre de Picard des revêtements doubles des surfaces algébriques. $C$. $R$. Acad. Sci. Paris Sér. I Math., 296(8):361-364, 1983.

[8] L. Costa and R. M. Miró-Roig. $G L(V)$-invariant Ulrich bundles on Grassmannians. Math. Ann., 361(1-2):443-457, 2015.

[9] Olivier Debarre. Higher-dimensional algebraic geometry. Universitext. Springer-Verlag, New York, 2001.

[10] David Eisenbud, Frank-Olaf Schreyer, and Jerzy Weyman. Resultants and Chow forms via exterior syzygies. J. Amer. Math. Soc., 16(3):537-579, 2003.

[11] Robert Friedman. Algebraic surfaces and holomorphic vector bundles. Universitext. Springer-Verlag, New York, 1998. 
[12] Ingo Hadan. Tangent conics at quartic surfaces and conics in quartic double solids. Math. Nachr., 210:127-162, 2000.

[13] Robin Hartshorne. Algebraic geometry. Springer-Verlag, New York-Heidelberg, 1977. Graduate Texts in Mathematics, No. 52.

[14] Robin Hartshorne. Stable reflexive sheaves. Math. Ann., 254(2):121-176, 1980.

[15] Allen Hatcher. Algebraic topology. Cambridge University Press, Cambridge, 2002.

[16] J. Herzog, B. Ulrich, and J. Backelin. Linear maximal Cohen-Macaulay modules over strict complete intersections. J. Pure Appl. Algebra, 71(2-3):187-202, 1991.

[17] Robert Lazarsfeld. Positivity in algebraic geometry. I, volume 48 of Ergebnisse der Mathematik und ihrer Grenzgebiete. 3. Folge. A Series of Modern Surveys in Mathematics [Results in Mathematics and Related Areas. 3rd Series. A Series of Modern Surveys in Mathematics]. Springer-Verlag, Berlin, 2004. Classical setting: line bundles and linear series.

[18] Renjie Lyu and Xuanyu Pan. Remarks on automorphism and cohomology of cyclic coverings, arXiv:1705.06618, 2017.

[19] John Milnor. Singular points of complex hypersurfaces. Annals of Mathematics Studies, No. 61. Princeton University Press, Princeton, N.J.; University of Tokyo Press, Tokyo, 1968.

School of Mathematics, Tata Institute of Fundamental Research, Mumbai - 400005.

Email address: param@math.tifr.res.in

School of Mathematics, Tata Institute of Fundamental Research, Mumbai - 400005, Current address: International Centre for Theoretical Sciences, Bengaluru - 560089.

Email address: poorna.narayanan@icts.res.in 